\title{
Two-Probe Setup with Improved Calibration for Online Impedance Measurement of Electrical Assets
}

This paper was downloaded from TechRxiv (https://www.techrxiv.org).

\section{LICENSE}

CC BY 4.0

SUBMISSION DATE / POSTED DATE

07-10-2021 / 19-10-2021

\section{CITATION}

Chou, Hui Lin (2021): Two-Probe Setup with Improved Calibration for Online Impedance Measurement of Electrical Assets. TechRxiv. Preprint. https://doi.org/10.36227/techrxiv.16755640.v1

DOI 


\title{
Two-Probe Setup with Improved Calibration for Online Impedance Measurement of Electrical Assets
}

\author{
Hui-Lin Chou
}

\begin{abstract}
The online impedance serves as one of the most crucial specification to evaluate the health status and efficiency of an electrical device or system. The inductive coupling technique is a preferred approach to measure the online impedance due to the ease of implementation of the circuit which has zero physical contact to the live electrical system. The existing inductive coupling method deployed to measure the online impedance of an electrical device under test (DUT) adopts two probes in total: an injecting inductive probe (IIP) and a receiving inductive probe (RIP). An open/short/load (OSL) calibration procedure is implemented to eradicate the ramifications of the probeto-probe coupling, however, based on the assumption that the calibration criterions (shorted, open and 50 $\Omega$ ) are approximated to their theoretical values in a specified frequency range. Hence, any measurement with frequency outside the specified range (i.e. larger than $1 \mathrm{MHz}$ ) will not be accurate due to the frequency-dependent residual inductances and capacitances of the calibration model. To overcome the aforesaid limitation, this paper introduces an improved calibration procedure which is applicable for a wider frequency range which takes the frequencydependent characteristics into consideration. With the two-probe measurement setup (TPMS), the adopted improved calibration procedure is introduced to eradicate the ramifications of the probe-to-probe coupling with the intention to refine the accuracy of the extracted online impedance.
\end{abstract}

\section{Introduction}

The online impedance serves as one of the most crucial specification to determine the health status and condition of an electrical system [1]. For example, the online impedance of the power grid has a consequential effect on the capability of the grid-connected power converters. Hence, inaccurate analysis of the online impedance can corrupt the performance and reliability of the grid-connected power converters [2-4]. In addition, the online impedance plays a significant role in the operation of an Induction Motor (IM) as early fault detection can prevent irreparable damage, reduce safety threats, minimize motor interruption, and the cost from reparation works and interruption $[5,6]$. Recognizing the significance of the online impedance of an electrical system, it is essential to establish a compelling method to extract the online impedance of an electrical system. Primarily, there are three means of approach in deriving the online impedances directly from electrical systems; being the voltage-current analysis method, the capacitive coupling method, and the inductive coupling method. Among the fore mentioned, the latter approach is a preferred method as it has zero physical contact to the live electrical system under test (SUT). Additionally, the clamp-on inductive probes utilised in this method can simply be secured and detached from the insulated cable that transmits power to the SUT which notably simplifies the implementation with minimal electrical risk [7].

The prevailing inductive coupling setup adopts two probes in total: an injecting inductive probe (IIP) and a receiving inductive probe (RIP), which is also known as the Two-Probe Measurement Setup (TPMS). An open/short/load (OSL) calibration procedure is implemented to eradicate any possible ramifications of the probeto-probe coupling [7], however, based on the assumption that the calibration criterions (shorted, open and 50 $\Omega$ ) are approximated to their theoretical values in a specified frequency range. Hence, any measurement with frequency outside the specified range (i.e. $\geq 1 \mathrm{MHz}$ ) will not be accurate due to the frequency-dependent residual inductances and capacitances of the calibration model.

The motivation of this paper is to introduce and adopt a more refined and reliable measurement setup to tackle the fore mentioned limitations of the existing measurement setup, thereby exploring new applications and improving the accuracy of the extracted online impedance. This paper is organized as follows: Section II reviews the literature of existing online impedance measurement methods. Section III presents the methods and principles adopted for the TPMS and discusses the existing OSL calibration procedure, improved calibration procedure and the proposed measurement configuration. Section IV discusses about the experimental results and its validation on the improved calibration procedure and proposed measurement configuration. Finally, Section V concludes this paper and proposes future works that are worthy of consideration.

\section{Section II. Literature Review of Online Impedance Measurement}

Taking into consideration the significance of the online impedance of electrical assets, the extraction of online impedance is now a notable process, thus, a few methods to extract the online impedance will be comprehensively 
examined with its integrity and limitations highlighted. The extraction methods can be categorized into three types, that is the voltage-current measurement method [8-13], capacitive coupling method [14-16] and lastly, the inductive coupling method [17-22].

\section{A. Voltage-Current Measurement Method}

The voltage-current measurement method is a direct method to obtain the online impedance. The online impedance of the DUT ( $\mathbf{Z}_{\text {DUT }}$ ) can be approximated through the extraction of the test signal voltage across the DUT and test signal current flowing through the DUT. It should be taken into account that the test signal may either be in the form of harmonics contributed by the DUT [8] or a signal generated by an external device, for example, a current transformer or a voltage transformer [23].

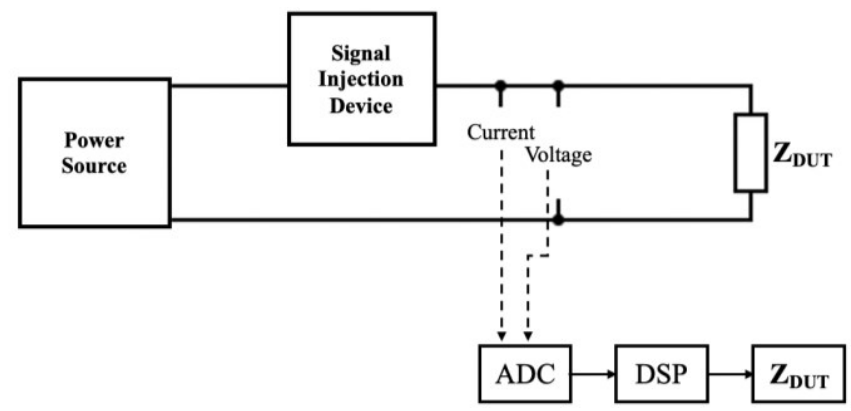

Figure 1. Fundamental setup of the voltage-current measurement method with an external signal injection device connected.

Figure 1 illustrates the fundamental setup of the voltage-current measurement method with an external signal injection device connected. The test signal will be injected into the wire connecting the power source and DUT. Thereafter, a voltage sensor and current sensor will then be used to extract the voltage across the DUT induced by the test signal and the induced current flowing through the DUT. Following, an analog-to-digital converter (ADC) is applied to process the analog voltage and current signals, followed by a digital signal processing (DSP) program to derive $\mathbf{Z}_{\text {DUT. }}$.

\section{B. Capacitive Coupling Method}

Figure 2 illustrates the fundamental setup of the capacitive coupling method to obtain the online impedance of an electrical DUT, indicated as $\mathbf{Z}_{\text {DUT. }}$. The DUT can be energized by either a DC or low-frequency alternating current (AC) power source, which is connected by a pair of inductors $\left(\mathrm{L}_{1}\right.$ and $\left.\mathrm{L}_{2}\right)$ and capacitors $\left(\mathrm{C}_{1}\right.$ and $\left.\mathrm{C}_{2}\right)$ to a measurement instrument, such as an impedance analyzer (IA) or a vector network analyzer (VNA) [14-16]. Capacitors $C_{1}$ and $C_{2}$ are included to prevent any form of power signal contributed by the power source from flowing into the measurement instrument meanwhile allowing high-frequency test signal generated by the measurement instrument to flow through the DUT.

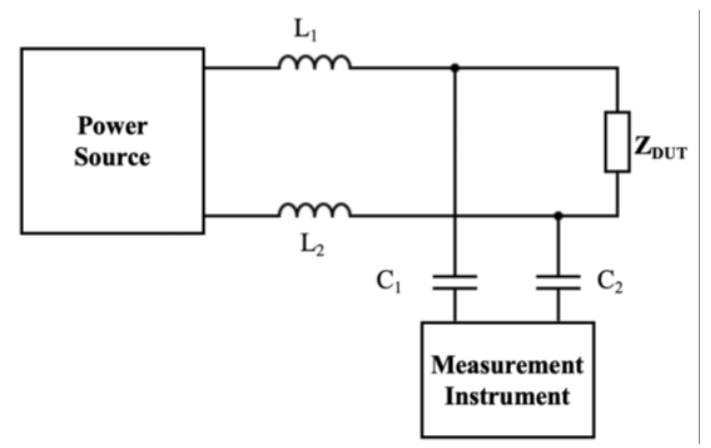

Figure 2. Fundamental setup of the capacitive coupling method.

Contrastingly, inductors $\mathrm{L}_{1}$ and $\mathrm{L}_{2}$ are included to serve as a blockage to prevent high-frequency test signal generated by the measurement instrument from flowing into the power source but they establish insignificant impedance characteristics for the low-frequency power signal. For DC or low-frequency AC power signal, capacitors $C_{1}$ and $C_{2}$ are treated as open switches as shown in Figure 3. Correspondingly, for high-frequency test 
signal, inductors $\mathrm{L}_{1}$ and $\mathrm{L}_{2}$ can be considered as open switches, and the corresponding impedances of the capacitors $\mathrm{C}_{1}$ and $\mathrm{C}_{2}$ are labelled as $\mathbf{Z}_{\mathbf{C} 1}$ and $\mathbf{Z}_{\mathbf{C} 2}$ respectively as shown in Figure 4.

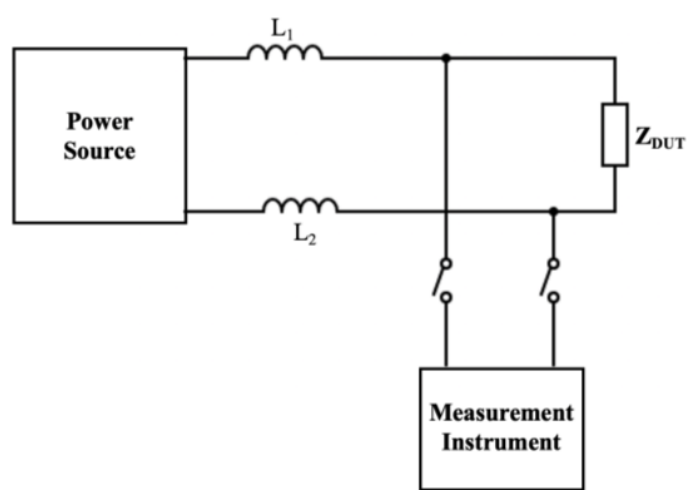

Figure 3. Capacitive coupling measurement setup for DC or low-frequency AC power signal.

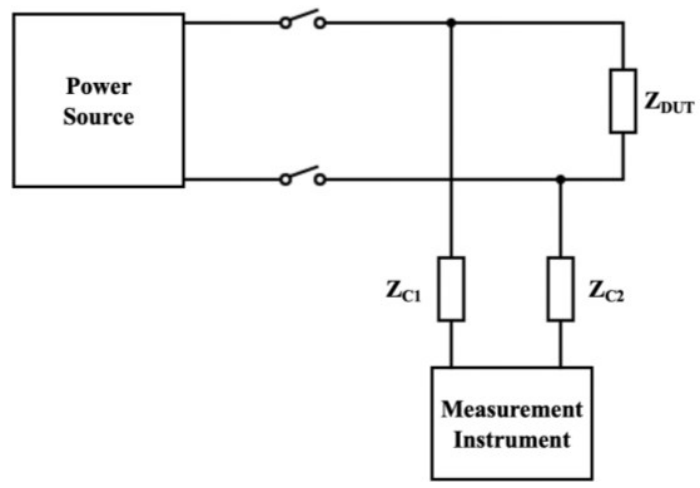

Figure 4. Capacitive coupling measurement setup for high-frequency test signal.

As illustrated in Figure 4, the overall equivalent impedance $\mathbf{Z}_{\text {Total }}$ can be measured straight with an IA, where $\mathbf{Z}_{\text {Total }}$ can be expressed as

$$
Z_{\text {Total }}=Z_{D U T}+Z_{C 1}+Z_{C 2}
$$

$\mathbf{Z}_{\text {DUT }}$ can then be calculated by de-embedding $\left(\mathbf{Z}_{\mathbf{C}_{1}}+\mathbf{Z}_{\mathbf{C} 2}\right)$ from the overall equivalent impedance $\mathbf{Z}_{\text {Total. }}$. On the other hand, if the VNA is being chosen as the measurement instrument [16], Figure 2 can be further organized as a two-port network circuit, as illustrated in Figure 5.

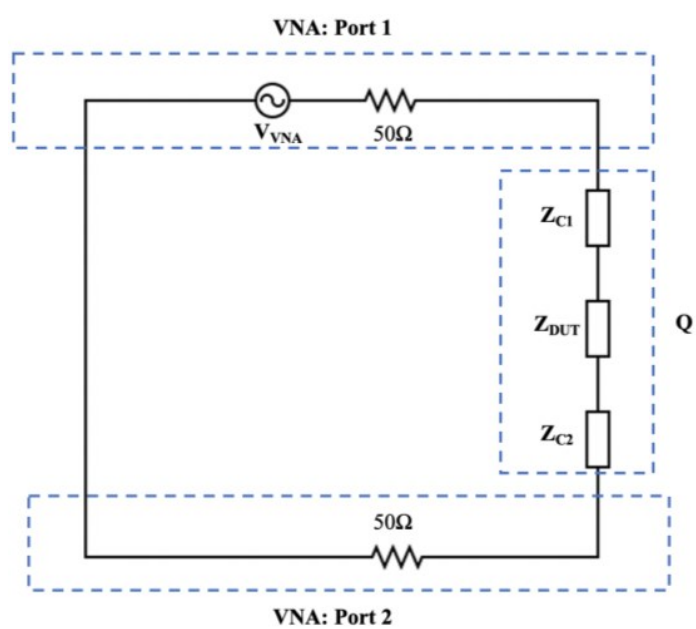

Figure 5. Equivalent two-port network circuit with respect to Figure 3.

As shown in Figure 5, the two-port network $\mathbf{Q}$ can be equated in terms of DUT, $\mathrm{C}_{1}$ and $\mathrm{C}_{2}$, where $\mathbf{Q}$ can be expressed corresponding to transmission $(\mathrm{ABCD})$ parameters. 


$$
Q=\left[\begin{array}{ll}
A & B \\
C & D
\end{array}\right]
$$

Since $\boldsymbol{B}=\boldsymbol{Z}_{\mathrm{DUT}}+\boldsymbol{Z}_{\mathrm{C} 1}+\boldsymbol{Z}_{\mathrm{C} 2}$, the scattering parameters (S-parameters) of $\mathbf{Q}$ can then be obtained straight with the VNA followed by the conversion of the S-parameters to $\boldsymbol{B}$ where [23]

$$
B=50 \times \frac{\left(1+S_{11}\right)\left(1+S_{22}\right)-S_{12} S_{21}}{2 S_{21}}
$$

The de-embedding of $\left(\mathbf{Z}_{\mathbf{C} 1}+\mathbf{Z}_{\mathbf{C} 2}\right)$ from $\boldsymbol{B}$ is then executed to obtain the value of $\mathbf{Z}_{\mathbf{D U T}}$.

\section{Inductive Coupling Method}

The initial findings on the inductive coupling method was published in [17]. The project was further built on and optimized to streamline the online impedance measurement procedure based on the cascaded two-port network theory $[18,19]$.

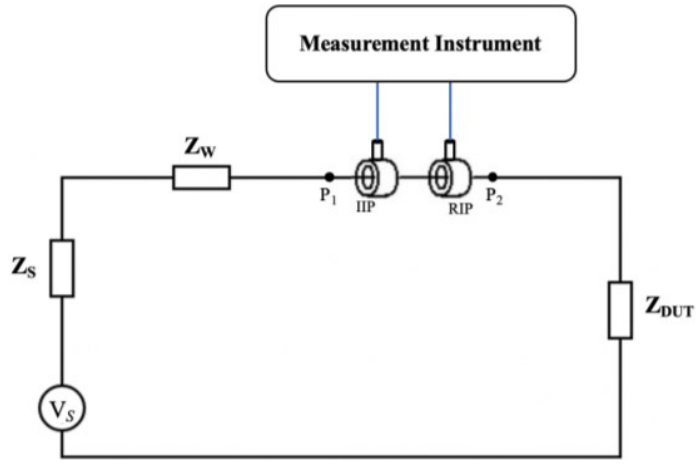

Figure 6. Two-Probe Measurement Setup (TPMS) for the inductive coupling method.

The inductively coupled measurement configuration to extract the online impedance of a DUT is shown in Figure 6 , where the DUT is energized by $\mathrm{V}_{\mathrm{S}}$ which can either be a DC source or a low-frequency AC power source via the wires connected to the DUT. A measurement instrument, IIP and RIP embodies the inductive coupling measurement setup, where the IIP functions as an injecting probe while the RIP functions as a receiving probe to receive the signal. Assuming that the computer-controlled signal generation and acquisition system (SGAS) is employed as the measurement instrument illustrated in Figure 2-6, a sweep-frequency test signal is generated and injected into the wire that serves as a connection between the source voltage $\left(\mathrm{V}_{\mathrm{S}}\right)$ and DUT, via channel 0 of the SGAS through the injecting probe. The response signal is then being detected by channel 1 of the SGAS via the receiving probe. The online impedance of the DUT is labelled as $\mathbf{Z}_{\mathbf{D U T}}$, whereas $\mathbf{Z}_{\mathbf{S}}$ and $\mathbf{Z}_{\mathbf{W}}$ denotes the impedance of the source voltage and circuitry wiring excluding the clamped sections of the wire, respectively [18]. The points where the wire are being clamped by the two inductive probes are denoted as $p_{1}$ and $p_{2}$ in Figure 6 . The equivalent impedance measured by the RIP can be expressed as

$$
Z_{X}=Z_{S}+Z_{W}+Z_{D U T}
$$

where $\mathbf{Z}_{\mathbf{X}}$ is the summation of the impedances contributed by the source voltage $\left(\mathbf{Z}_{S}\right)$, wiring connection excluding the clamped section $\left(\boldsymbol{Z}_{\boldsymbol{W}}\right)$ and DUT $\left(\boldsymbol{Z}_{\boldsymbol{D U}}\right)$. Developed upon the idea of cascaded two-port networks, Figure 7 illustrates the equivalent circuit model of Figure 6 , where $Z_{S G C}$ is the corresponding impedance of the signal generation card (SGC) of the SGAS and $\mathrm{Z}_{\mathrm{CH}}$ and $\mathrm{Z}_{\mathrm{CH} 1}$ are the respective input impedances of channel 0 and 1 of the signal acquisition card (SAC) of the SGAS. As illustrated in Figure 7, the section of the wire where is being clamped by the IIP can be devised as a two-port ABCD matrix, denoted $\mathbf{Q}_{\mathbf{I}}$, where one port serves as the input terminal of the IIP and the other serves the two terminals of the wire being clamped. Correspondingly, the section where the wire is being clamped by the RIP can be devised as a two-port ABCD matrix as well, denoted $\mathbf{Q}_{\mathbf{R}}$, where one port serves as the output terminal of the RIP and the other serves the two terminals of the wire being clamped. Moreover, $\mathbf{Q x}$ represents the two-port ABCD matrix for the resultant impedance of the measuring setup $\left(\mathbf{Z}_{\mathbf{X}}\right)$. To measure the value of $\mathbf{Z}_{\mathbf{x}}$, the SGC of the SGAS generates a sinusoidal test signal of a pre-determined amplitude $\left(\mathrm{V}_{\mathrm{SGC}}\right)$ and frequency $\left(f_{\text {sig }}\right)$ to be injected into the circuit. It is worth noting that the resultant impedance is extracted at $f_{\text {sig }}$ ranging from a few $\mathrm{kHz}$ to a few $\mathrm{MHz}$. Given that $f_{\text {sig }}$ is significantly larger compared to the frequency of the source voltage $\left(\mathrm{V}_{\mathrm{s}}\right)$, whereby DC $(0 \mathrm{~Hz})$ or low-frequency AC power source $(50 \sim 60 \mathrm{~Hz}), \mathrm{V}_{\mathrm{s}}$ can then be considered as $\mathrm{AC}$ short at $f_{\text {sig. }}$. 


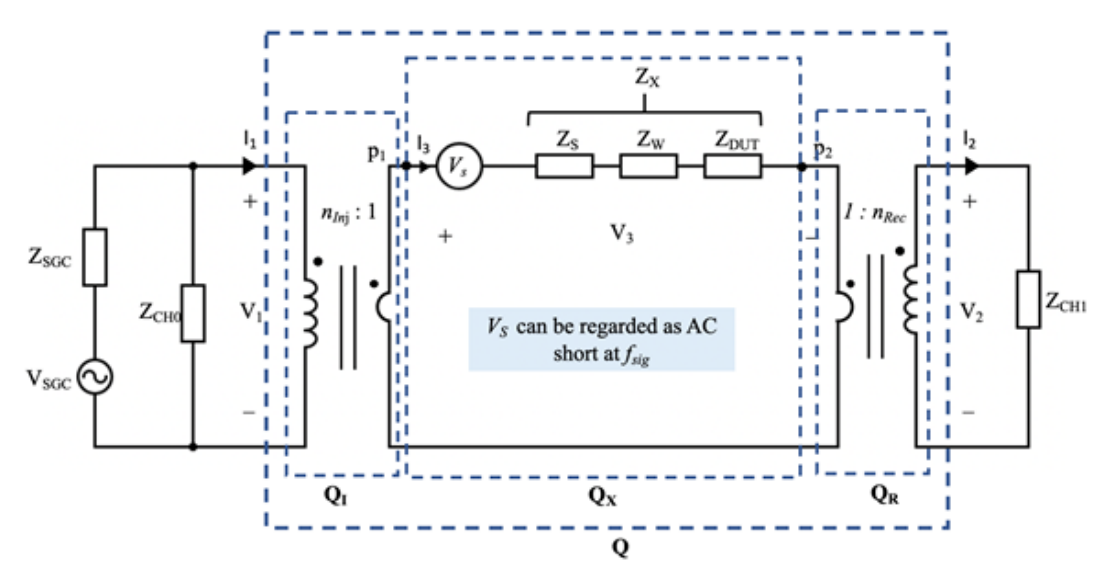

Figure 7. Equivalent circuit of Figure 6 depicted through cascaded two-port network.

Considering the three dual-port networks $\mathbf{Q}_{\mathbf{I}}, \mathbf{Q}_{\mathbf{R}}$ and $\mathbf{Q}_{\mathbf{x}}$ are arranged in a cascaded manner, the resultant dualport network $\mathbf{Q}$ can be equated as

$$
\mathbf{Q}=\mathbf{Q}_{I} \mathbf{Q}_{X} \mathbf{Q}_{R}
$$

Thence, the three dual-port networks can be expressed with reference to their corresponding transmission (ABCD) parameters

$$
\left[\begin{array}{ll}
A & B \\
C & D
\end{array}\right]=\left[\begin{array}{ll}
A_{I} & B_{I} \\
C_{I} & D_{I}
\end{array}\right]\left[\begin{array}{ll}
A_{X} & B_{X} \\
C_{X} & D_{X}
\end{array}\right]\left[\begin{array}{ll}
A_{R} & B_{R} \\
C_{R} & D_{R}
\end{array}\right]
$$

By solving $\mathbf{Q}_{\mathbf{X}}, \mathbf{Z}_{\mathbf{X}}$ can be determined as $\boldsymbol{B}_{\boldsymbol{X}}=\boldsymbol{Z}_{\boldsymbol{X}}$ [24]. $\mathbf{Q}_{\mathbf{X}}$ can then be calculated following the values of $\mathbf{Q}_{\text {, }} \mathbf{Q}_{\mathbf{I}}$ and $\mathbf{Q}_{\mathbf{R}}$ being known. The value of $\mathbf{Q}$ can be obtained straight from the measuring instrument, in this case, the SGAS, in Figure 6. With the measured equivalent impedance $\mathbf{Z}_{\mathbf{x}}$, $\mathbf{Z}_{\text {DUT }}$ can then be calculated by de-embedding $\left(\mathbf{Z}_{\mathbf{S}}+\mathbf{Z}_{\mathbf{w}}\right)$ from $\mathbf{Z}_{\mathbf{x}}$. $\left(\mathbf{Z}_{\mathbf{S}}+\mathbf{Z}_{\mathbf{w}}\right)$ can be pre-established by using a capacitor of appropriate rating to bypass the DUT which gives an AC short circuit to the test signal. In real circumstances, the magnitude of $\mathbf{Z}_{\text {Dut }}$ is usually much larger than the magnitude of $\left(\mathbf{Z}_{\mathbf{s}}+\mathbf{Z}_{\mathbf{w}}\right)$. This section explains and highlights the literature review of the inductive coupling method, whereas a more comprehensive explanation on the configuration and methodology of the inductive coupling method will be discussed in the next chapter.

In summary, this chapter studies and analyses three widely practiced online impedance extraction methods; that is the voltage-current measurement method, capacitive coupling method and finally, the inductive coupling method. Out of the three fore mentioned methods, the inductive coupling method surpassed the other two methods in terms of its non-intrusive nature and ease of implementation. To deduce the online impedance, the voltage sensor that constitutes the voltage-current measurement setup and the pair of capacitors that constitute the capacitive coupling method will inevitably require physical contact to the live DUT and hence, increasing the threat posed by electrical hazards. Additionally, for a live DUT powered with a high voltage level, the voltage sensor and the pair of capacitors from the voltage-current measurement method and capacitive coupling method respectively, are strained with considerable stresses (e.g. thermal), which in turn account for consistent maintenance and renewal; which may result in redundant downtime of the DUT. Despite its dominance in measuring the online impedance, the inductive coupling method still inevitably encounter some drawbacks that need to be improved on. This paper will address these drawbacks and explain in detail on how the inductive coupling method can be further developed. The drawbacks are outlined as follows: the capability to eliminate any ramifications generated by the coupling between the two inductive probes and the competency to obtain the online impedance of DUTs or electrical devices where electrical noise and power surges are considerably notable.

\section{Section III. Methods and Principles}

\section{A. Basic Setup with Two Inductive Probes}

The inductive coupling method utilizes a two-probe measurement setup (TPMS) concept. Figure 8 illustrates the basic measurement configuration to extract the online impedance of a DUT, whereby a DC power source or lowfrequency AC power source is being used to energize the DUT via connecting wires. 


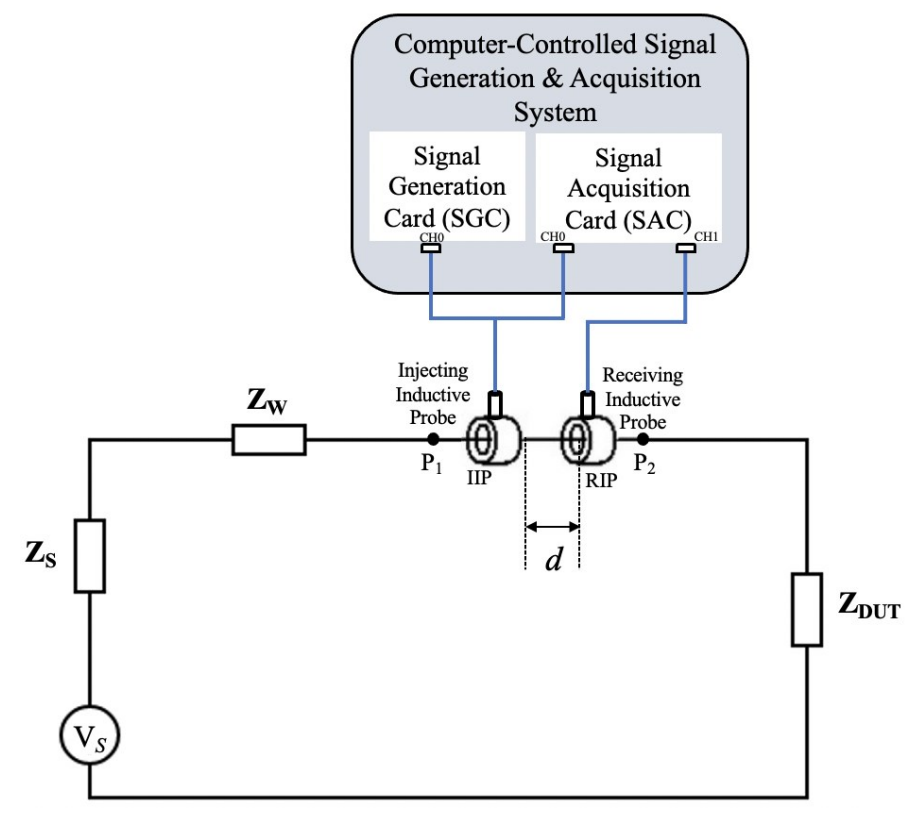

Figure 8. Basic measurement configuration for the inductive coupling method.

The computer-controlled signal generation and acquisition system (SGAS), injecting inductive probe (IIP) and receiving inductive probe (RIP) embodies the measurement configuration of the inductive coupling approach to extract the online impedance of the DUT $\left(\mathbf{Z}_{\mathbf{D U T}}\right)$. The computer-controlled SGAS comes with built in signal generation card (SGC) and signal acquisition card (SAC) integrated together. The SGC then generates a sinusoidal test waveform of a pre-determined frequency $\left(f_{\text {sig }}\right)$. The sinusoidal test signal travels from channel 0 of the SGC through the IIP via the connected wire which lies between the source voltage $\left(\mathrm{V}_{\mathrm{S}}\right)$ and DUT, meanwhile the RIP processes the feedback signal at the position where the two probes are clamped at ( $\mathrm{p}_{1}$ and $\left.\mathrm{p}_{2}\right)$. The voltage of the test signal at the input terminal and voltage of the feedback signal at the output terminal of the IIP is then being processed by channel $0(\mathrm{CH} 0)$ and channel $1(\mathrm{CH} 1)$ of the signal acquisition card $(\mathrm{SAC})$ within the SGAS, respectively. The impedance contributed by the source voltage and electrical wirings excluding the sections that are being clamped by the probes of the measurement setup is denoted as $\mathbf{Z}_{\mathbf{S}}$ and $\mathbf{Z}_{\mathbf{W}}$ respectively, as shown in Figure 8 [25]. The equivalent impedance measured by the two probes can be represented by $\mathbf{Z}_{\mathbf{x}}$, where

$$
Z_{X}=Z_{S}+Z_{W}+Z_{D U T}
$$

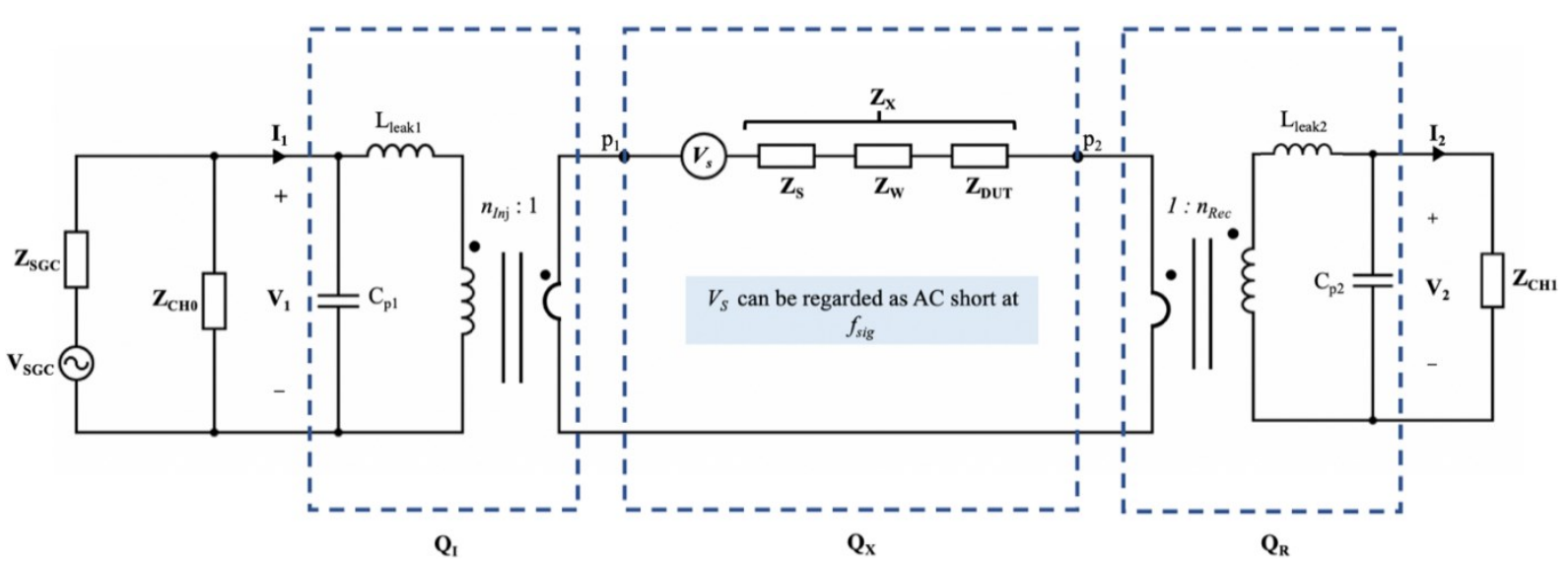

Figure 9. Inductive Coupling Method represented as a cascaded two-port network model.

Figure 9 illustrates the cascaded dual-port network model of the inductive coupling method. The voltage and internal impedance at the $\mathrm{SGC}$ are denoted as $\mathbf{V}_{\mathbf{S G C}}$ and $\mathbf{Z}_{\mathbf{S G C}}$ respectively, while $\mathbf{Z}_{\mathbf{C H} \mathbf{0}}$ and $\mathbf{Z}_{\mathbf{C H 1}}$ represent the input impedances of $\mathrm{CH} 0$ and $\mathrm{CH} 1$ of the $\mathrm{SAC}$, respectively. The equivalent voltage and current of the sinusoidal signal at the input terminal of the IIP are denoted by $\mathbf{V}_{\mathbf{1}}$ and $\mathbf{I}_{\mathbf{1}}$, respectively; $\mathbf{V}_{\mathbf{2}}$ and $\mathbf{I}_{\mathbf{2}}$ represents the voltage and current of the sinusoidal signal at the output terminal of the RIP respectively. $\mathbf{Q}_{I}$ represents the two-port network of the IIP which includes the segment of the wire that has been clamped by the IIP, where $\mathrm{L}_{\text {Leak } 1}$ and $\mathrm{C}_{\mathrm{p} 1}$ denote the 
leakage inductance and parasitical capacitance at the IIP side, respectively. $\mathbf{Q}_{\mathbf{R}}$ represents the two-port network at the RIP side which includes the segment of the wire that has been clamped by the RIP, where $\mathrm{L}_{\text {Leak } 2}$ and $\mathrm{C}_{\mathrm{p} 2}$ denote the leakage inductance and parasitical capacitance at the RIP side, respectively. Qx represents the dual-port network of the equivalent impedance $\left(\mathbf{Z}_{\mathbf{x}}\right)$ measured by the two probes. In consideration of the significantly high frequency of the generated sinusoidal test signal compared to the frequency of the source voltage (Vs), Vs can be considered as an $\mathrm{AC}$ short. Interpreting the three fore mentioned two-port networks $\left(\mathbf{Q}_{\mathbf{I}}, \mathbf{Q}_{\mathbf{R}}\right.$ and $\left.\mathbf{Q}_{\mathbf{x}}\right)$ in accordance with their corresponding ABCD parameters, the correlation of the input terminal of the IIP and the output terminal of the RIP can be expressed as

$$
\left[\begin{array}{l}
V_{1} \\
I_{1}
\end{array}\right]=\left[\begin{array}{ll}
A_{I} & B_{I} \\
C_{I} & D_{I}
\end{array}\right]\left[\begin{array}{ll}
A_{X} & B_{X} \\
C_{X} & D_{X}
\end{array}\right]\left[\begin{array}{ll}
A_{R} & B_{R} \\
C_{R} & D_{R}
\end{array}\right]\left[\begin{array}{l}
V_{2} \\
I_{2}
\end{array}\right]
$$

Given that $\boldsymbol{I}_{2}=\frac{V_{2}}{Z_{C H 1}}$ and $\mathbf{Q} \mathbf{x}$ can be equated as [24]

$$
\boldsymbol{Q}_{X}=\left[\begin{array}{cc}
1 & Z_{X} \\
0 & 1
\end{array}\right]
$$

$\mathbf{Z}_{\mathbf{x}}$ can be worked out by

$$
Z_{X}=\frac{1}{A_{I}\left(C_{R}+\frac{D_{R}}{Z_{C H 1}}\right)} \cdot \frac{V_{1}}{V_{2}}-\frac{A_{R}+\frac{B_{R}}{Z_{C H 1}}}{C_{R}+\frac{D_{R}}{Z_{C H 1}}}-\frac{B_{I}}{A_{I}}
$$

$\mathbf{Z}_{\mathbf{C H 1}}$ can be retrieved from the datasheet of the SGC. Thence, $\mathbf{Z}_{\mathbf{X}}$ can be determined through the computation of $\mathbf{V}_{\mathbf{1}}$ and $\mathbf{V}_{\mathbf{2}}$. Immediately after $\mathbf{Z}_{\mathbf{X}}$ is determined, $\mathbf{Z}_{\text {Dut }}$ can be approximated by de-embedding $\left(\mathbf{Z}_{\mathbf{S}}+\mathbf{Z}_{\mathbf{W}}\right)$ from $\mathbf{Z}_{\mathbf{X}}$.

\section{B. Open/Short/Load Calibration}

The inductive coupling method is a preferred technique to extract the online impedance of an electrical asset due to its non-intrusive feature. However, in real circumstances where space scarcity is a factor to be concerned of, the injecting and receiving probes connected to the measurement setup will be closely spaced thus inducing probeto-probe coupling which may produce undesirable effects on the measurement procedure and cannot be overlooked. Thus, the open/short/load (OSL) calibration procedure is implemented to eliminate the ramifications of the coupling between the two inductive probes with the intent to eliminate any error that may compromise the accuracy and precision of the measurement.

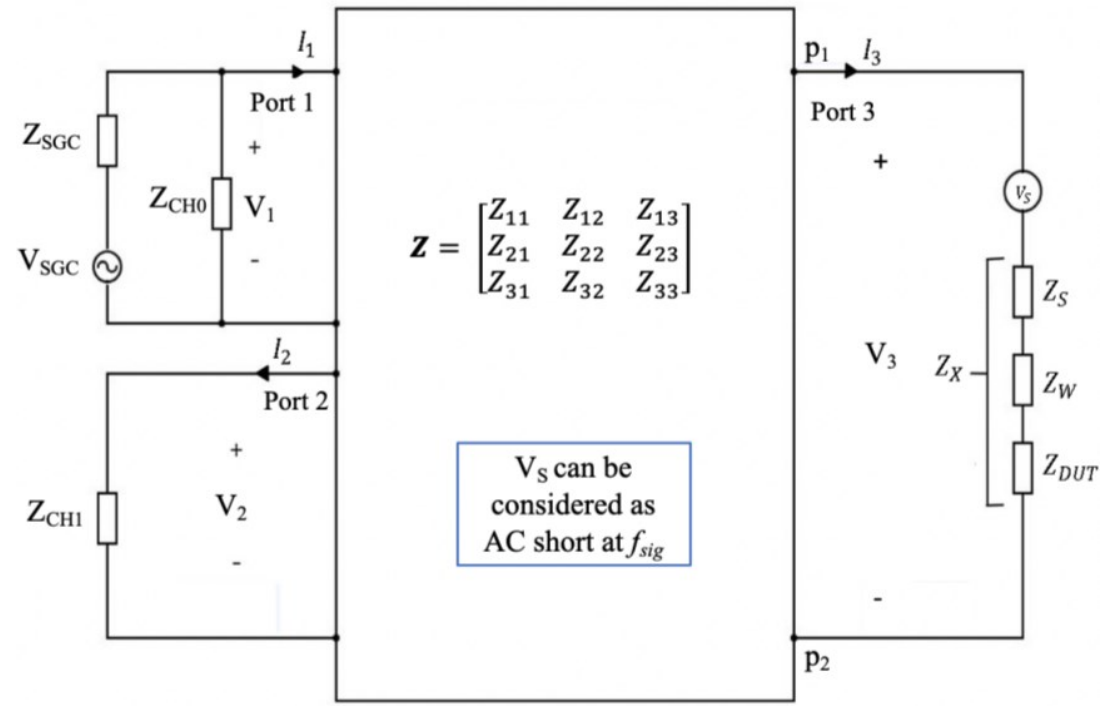

Figure 10. Three-port network depiction of Figure 8.

From Figure 10, $Z_{\mathrm{SGC}}$ is the equivalent impedance of the $\mathrm{SGC} ; \mathrm{Z}_{\mathrm{CH} 0}$ and $\mathrm{Z}_{\mathrm{CH} 1}$ are the corresponding input impedances of $\mathrm{CH} 0$ and $\mathrm{CH} 1$ of the $\mathrm{SAC}$. $\mathrm{V}_{1}$ and $\mathrm{I}_{1}$ denotes the equivalent voltage and current of the sinusoidal test signal generated by the SGC at the input terminal of the IIP (Port 1), respectively. $\mathrm{V}_{2}$ and $\mathrm{I}_{2}$ denotes the equivalent voltage and current at the output terminal of the RIP (Port 2) while $\mathrm{V}_{3}$ and $\mathrm{I}_{3}$ are the voltage and current produced at the input terminal (Port 3 ) of the resultant impedance $\left(Z_{X}\right)$ to be measured. As mentioned earlier, in consideration of the significantly high frequency of the generated sinusoidal test signal $\left(f_{\text {sig }}\right)$ compared to the 
frequency of the source voltage $\left(\mathrm{V}_{\mathrm{S}}\right), \mathrm{V}_{\mathrm{S}}$ can be considered as an $\mathrm{AC}$ short. Hence, the induced coupling between the two probes, also known as the "probe-to-probe coupling" can be expressed in terms of an impedance matrix given by

$$
\boldsymbol{Z}=\left[\begin{array}{lll}
Z_{11} & Z_{12} & Z_{13} \\
Z_{21} & Z_{22} & Z_{23} \\
Z_{31} & Z_{32} & Z_{33}
\end{array}\right]
$$

The correlation of the corresponding voltages and currents of each ports can be expressed by

$$
\left[\begin{array}{l}
\boldsymbol{V}_{\mathbf{1}} \\
\boldsymbol{V}_{\mathbf{2}} \\
\boldsymbol{V}_{\mathbf{3}}
\end{array}\right]=\left[\begin{array}{lll}
Z_{11} & Z_{12} & Z_{13} \\
Z_{21} & Z_{22} & Z_{23} \\
Z_{31} & Z_{32} & Z_{33}
\end{array}\right] \cdot\left[\begin{array}{l}
\boldsymbol{I}_{\mathbf{1}} \\
\boldsymbol{I}_{\mathbf{2}} \\
\boldsymbol{I}_{\mathbf{3}}
\end{array}\right]
$$

Given $\boldsymbol{I}_{2}=\frac{V_{2}}{Z_{C H 1}}$ and $\boldsymbol{I}_{3}=\frac{V_{3}}{Z_{X}}$, the above equation can be re-written as

$$
\left[\begin{array}{l}
\boldsymbol{V}_{\mathbf{1}} \\
\boldsymbol{V}_{\mathbf{2}} \\
\boldsymbol{V}_{\mathbf{3}}
\end{array}\right]=\left[\begin{array}{lll}
Z_{11} & Z_{12} & Z_{13} \\
Z_{21} & Z_{22} & Z_{23} \\
Z_{31} & Z_{32} & Z_{33}
\end{array}\right] \cdot\left[\begin{array}{c}
\boldsymbol{I}_{\mathbf{1}} \\
\boldsymbol{V}_{\mathbf{2}} / Z_{C H 1} \\
\boldsymbol{V}_{\mathbf{3}} / \boldsymbol{Z}_{\boldsymbol{X}}
\end{array}\right]
$$

By dividing $\mathbf{V}_{2}$ throughout the above equation on both sides,

$$
\left[\begin{array}{c}
\boldsymbol{V}_{\mathbf{1}} / \boldsymbol{V}_{\mathbf{2}} \\
1 \\
\boldsymbol{V}_{\mathbf{3}} / \boldsymbol{V}_{\mathbf{2}}
\end{array}\right]=\left[\begin{array}{lll}
Z_{11} & Z_{12} & Z_{13} \\
Z_{21} & Z_{22} & Z_{23} \\
Z_{31} & Z_{32} & Z_{33}
\end{array}\right] \cdot\left[\begin{array}{c}
\boldsymbol{I}_{\mathbf{1}} / \boldsymbol{V}_{\mathbf{2}} \\
1 / Z_{C H 1} \\
\left(\boldsymbol{V}_{\mathbf{3}} / \boldsymbol{Z}_{\boldsymbol{X}}\right) / \boldsymbol{V}_{\mathbf{2}}
\end{array}\right]
$$

By solving the equation, $\frac{V_{1}}{V_{2}}, \frac{V_{3}}{V_{2}}$ and $\frac{I_{1}}{V_{2}}$ can be expressed with reference to the three-port impedance parameters from matrix $\mathbf{Z}, \mathbf{Z}_{\mathrm{CH} 1}$ and $\mathbf{Z} \mathbf{x}$, where $\mathbf{V}_{\mathbf{1}} / \mathbf{V}_{\mathbf{2}}$ can be equated as

$$
\frac{v_{1}}{V_{2}}=\frac{a_{1} \cdot Z_{X}+a_{2}}{Z_{X}+a_{3}}
$$

Where the detailed derivation is shown in the Appendices. In addition, the comprehensive expressions of $\mathrm{a}_{1}, \mathrm{a}_{2}$ and $\mathrm{a}_{3}$ are also included in the appendices. Eventually, $\mathbf{Z} \mathbf{x}$ can be expressed in terms of $\mathbf{V}_{\mathbf{1}} / \mathbf{V}_{\mathbf{2}}$ as follows

$$
Z_{X}=\frac{a_{3} \cdot \frac{V_{1}}{V_{\mathbf{2}}}-a_{2}}{-\frac{V_{\mathbf{1}}}{V_{\mathbf{2}}}+a_{1}}
$$

$\mathbf{Z}_{\mathbf{x}}$ can be obtained through $\frac{\boldsymbol{V}_{\mathbf{1}}}{\boldsymbol{V}_{\mathbf{2}}}$ once $\mathrm{a}_{1}, \mathrm{a}_{2}$ and $\mathrm{a}_{3}$ are made known, taking into account that $\mathbf{V}_{\mathbf{1}}$ and $\mathbf{V}_{\mathbf{2}}$ can be obtained straight from the measurement setup in Figure 8. In consideration of a SAC with a unique $Z_{\mathrm{CH}}$, the values of $\mathrm{a}_{1}, \mathrm{a}_{2}$ and $\mathrm{a}_{3}$ are dependent on the impedance parameters of $\mathbf{Z}$ (i.e. $\mathbf{Z}_{\mathrm{ij}}$ ) where i,j $=1,2,3$. The three-port impedance parameters are influenced by the physical characteristics of the inductive probes and sinusoidal test signal frequency $\left(f_{\text {sig }}\right)$. In addition, the distance between the two inductive probes, denoted as $d$ in Figure 8, also has an influence on the three-port impedance parameters as the probe-to-probe coupling fluctuates with the distance between the two inductive probes. To obtain the values of $\mathrm{a}_{1}, \mathrm{a}_{2}$ and $\mathrm{a}_{3}$, the resultant impedance $\left(\mathbf{Z}_{\mathbf{X}}\right)$ can be substituted with three pre-determined specifications before executing the actual measurement. The three prevailing calibration standards used by industries are open, short and $50 \Omega$ resistive load, in which this project also adopts the exact same calibration criterions. When Port 3 in Figure 10 is open-circuited, $\mathbf{Z}_{\mathbf{X}}$ can be considered as $\infty$ and this criterion is denoted as $R_{\infty}$ where

$$
a_{1}=\left.\frac{V_{1}}{V_{2}}\right|_{R_{\infty}}
$$

When Port 3 in Figure 10 is shorted, $\mathbf{Z} \mathbf{x}$ can be considered as $0 \Omega$ and this criterion is denoted as $\mathrm{R}_{0}$ where

$$
a_{2}=\left.a_{3} \cdot \frac{v_{1}}{V_{2}}\right|_{R_{0}}
$$

Lastly, when Port 3 in Figure 10 is terminated with a $50 \Omega$ resistive load, $\mathbf{Z}_{\mathbf{x}}$ can be considered as $50 \Omega$ and this criterion is denoted as $R_{50}$, and it can be expressed as 


$$
R_{50}=\frac{\left.a_{3} \cdot \frac{V_{\mathbf{1}}}{V_{\mathbf{2}}}\right|_{R_{50}}-a_{2}}{-\left.\frac{V_{\mathbf{1}}}{V_{\mathbf{2}}}\right|_{R_{50}}+a_{1}}
$$

In the above expressions of $\mathrm{a}_{1}, \mathrm{a}_{2}$ and $\mathrm{R}_{50}, \frac{\boldsymbol{V}_{\mathbf{1}}}{\boldsymbol{V}_{\mathbf{2}}} \mathrm{I}_{c}$ represents the voltage ratio at the input terminal of the IIP and the output terminal of the RIP, where " $c$ " denotes the criterion selected, either $R_{\infty}, R_{0}$ or $R_{50}$. In view of the fact that $\left.\frac{V_{1}}{V_{2}}\right|_{c}$ can be determined via the measurement setup, $a_{2}$ and $a_{3}$ can then be obtained through the following expressions, respectively,

$$
\begin{gathered}
a_{2}=\left.R_{50} \cdot \frac{V_{1}}{V_{2}}\right|_{R_{0}} \cdot \frac{\left(\left.\frac{V_{1}}{V_{2}}\right|_{R_{\infty}}-\left.\frac{V_{1}}{V_{2}}\right|_{R_{50}}\right)}{\left(\left.\frac{V_{1}}{V_{2}}\right|_{R_{50}}-\left.\frac{V_{1}}{V_{2}}\right|_{R_{0}}\right)} \\
a_{3}=R_{50} \cdot \frac{\left(\left.\frac{V_{1}}{V_{2}}\right|_{R_{\infty}}-\left.\frac{V_{1}}{V_{2}}\right|_{R_{50}}\right)}{\left(\left.\frac{V_{1}}{V_{2}}\right|_{R_{50}}-\left.\frac{V_{1}}{V_{2}}\right|_{R_{0}}\right)}
\end{gathered}
$$

Thence, $\mathbf{Z}_{\mathbf{X}}$ can be derived through the expression as follows

$$
Z_{X}=R_{50} \cdot \frac{\left(\left.\frac{V_{1}}{V_{2}}\right|_{R_{\infty}}-\left.\frac{V_{1}}{V_{2}}\right|_{R_{50}}\right)\left(\frac{V_{1}}{V_{2}}-\left.\frac{V_{1}}{V_{2}}\right|_{R_{0}}\right)}{\left(\left.\frac{V_{1}}{V_{2}}\right|_{R_{50}}-\left.\frac{V_{1}}{V_{2}}\right|_{R_{0}}\right)\left(\left.\frac{V_{1}}{V_{2}}\right|_{R_{\infty}}-\frac{V_{1}}{V_{2}}\right)}
$$

However, it should be taken into consideration that the prerequisite of deriving $\mathbf{Z}_{\mathbf{X}}$ from the above expression requires $\left.\mathrm{V}_{1}\right|_{\mathrm{c}}$ and $\left.\mathrm{V}_{2}\right|_{\mathrm{c}}$ to be determinable. Considering $\left.\mathrm{V}_{1}\right|_{\mathrm{c}}$ is the signal at the input terminal of the IIP, the signalto-noise-ratio (SNR) should be satisfactory given that the signal should exceed the measurement system's noise floor. Under the criterion of $\boldsymbol{Z}_{\boldsymbol{X}}=\infty$, if the probe-to-probe coupling is strong, $\left.V_{2}\right|_{R_{\infty}}$ is still determinable, thus $\mathrm{Z}_{\mathrm{X}}$ can still be measured. If the coupling between the two probes is poor, $\left.V_{2}\right|_{R_{\infty}}$ will fall below the measurement system's noise floor which is too insignificant to influence the measurement and thus negligible. Hence, the influence of the coupling between the two inductive probes on the precision of the measured online impedance $\mathbf{Z}_{\mathbf{X}}$ is insignificant thus negligible, and $\mathbf{Z}_{\mathbf{X}}$ can be accurately derived through

$$
Z_{X}=\frac{1}{A_{I}\left(C_{R}+\frac{D_{R}}{Z_{C H 1}}\right)} \cdot \frac{V_{1}}{V_{2}}-\frac{A_{R}+\frac{B_{R}}{Z_{C H 1}}}{C_{R}+\frac{D_{R}}{Z_{C H 1}}}-\frac{B_{I}}{A_{I}}
$$

To simplify the above expression, it can be revised as

$$
Z_{X}=k \cdot \frac{v_{1}}{V_{2}}+b
$$

Where

$$
\begin{gathered}
k=\frac{1}{A_{I}\left(C_{R}+\frac{D_{R}}{Z_{C H 1}}\right)} \\
b=-\frac{A_{R}+\frac{B_{R}}{Z_{C H 1}}}{C_{R}+\frac{D_{R}}{Z_{C H 1}}}-\frac{B_{I}}{A_{I}}
\end{gathered}
$$

The introduced calibration procedure can also be employed to obtain the values of $\mathrm{k}$ and $\mathrm{b}$. When $\mathbf{Z}_{\mathbf{x}}$ is terminated with a short-circuit, where $\mathbf{Z}_{\mathbf{X}}=0 \Omega, \mathbf{Z}_{\mathbf{X}}$ can be expressed as

$$
0=\left.k \cdot \frac{V_{1}}{V_{2}}\right|_{R_{0}}+b
$$

Likewise, when $\mathbf{Z}_{\mathbf{x}}$ is terminated with a $50 \Omega$ resistive load, where $\mathbf{Z}_{\mathbf{x}}=\mathrm{R}_{50}, \mathbf{Z}_{\mathbf{x}}$ can be expressed as

$$
R_{50}=\left.k \cdot \frac{V_{1}}{V_{2}}\right|_{R_{50}}+b
$$

By solving the two equations above, $\mathrm{k}$ and $\mathrm{b}$ can be determined by

$$
k=\frac{R_{50}}{\left(\left.\frac{V_{1}}{V_{2}}\right|_{R_{50}}-\left.\frac{V_{1}}{V_{2}}\right|_{R_{0}}\right)}
$$




$$
b=\frac{-\left.R_{50} \cdot \frac{V_{\mathbf{1}}}{V_{\mathbf{2}}}\right|_{R_{0}}}{\left(\left.\frac{V_{\mathbf{1}}}{V_{\mathbf{2}}}\right|_{R_{50}}-\left.\frac{V_{\mathbf{1}}}{V_{\mathbf{2}}}\right|_{R_{0}}\right)}
$$

Hence, $\mathbf{Z}_{\mathbf{X}}$ can ultimately be obtained by

$$
Z_{X}=\frac{R_{50} \cdot\left(\frac{V_{1}}{V_{2}}-\left.\frac{V_{1}}{V_{2}}\right|_{R_{0}}\right)}{\left(\left.\frac{V_{1}}{V_{2}}\right|_{R_{50}}-\left.\frac{V_{1}}{V_{2}}\right|_{R_{0}}\right)}
$$

On the flip side, the OSL calibration procedure is based upon the assumption that the calibration criterions (shorted, open and $50 \Omega$ resistive load) are approximated to their theoretical values in a specified frequency range. Hence, any measurement with frequency outside the specified range (i.e. $\geq 1 \mathrm{MHz}$ ) will not be accurate due to the frequency-dependent residual inductances and capacitances of the calibration model.

\section{Proposed Improved Calibration Procedure}

To overcome the aforesaid drawback, an improved calibration procedure will be proposed and comprehensively discussed in this section. By executing the improved calibration procedure, the coupling between the two inductive probes can be carefully assessed and have its ramifications be eliminated. As mentioned in the above section, the three calibration criterions adopted by the OSL calibration model are required to successfully devise a shorted, open and $50 \Omega$ resistive load termination at the input terminal (Port 3) of $\mathbf{Z}$ as seen in Figure 10. Likewise, to work out the values of $a_{1}, a_{2}$ and $a_{3}$ of a designated TPMS with unique $Z_{\mathrm{CH} 1}$ and $\mathbf{Z}$, the proposed improved calibration procedure also requires three distinguishable calibration criterions, denoted by $\mathrm{CC} 1, \mathrm{CC} 2$ and $\mathrm{CC} 3$. In consequence of the frequency-dependent characteristics of $\mathrm{CC} 1, \mathrm{CC} 2$ and $\mathrm{CC} 3$, their respective frequencydependent impedances $\left(\mathbf{Z}_{\mathbf{C C} 1}, \mathbf{Z}_{\mathbf{C C} 2}\right.$ and $\mathbf{Z}_{\mathbf{C C} \text { 3 }}$, where $\left.\mathbf{Z}_{\mathbf{C C} \mathbf{1}} \neq \mathbf{Z}_{\mathbf{C C} \mathbf{2}} \neq \mathbf{Z}_{\mathbf{C C} \text { 3 }}\right)$ can be obtained by utilizing an impedance analyzer prior the calibration procedure. With reference to

$$
Z_{X}=\frac{a_{3} \cdot \frac{V_{1}}{V_{2}}-a_{2}}{-\frac{V_{1}}{V_{2}}+a_{1}}
$$

When the input terminal (Port 3) of $\mathbf{Z}$ in Figure 10 is being terminated with $\mathrm{CC} 1, \mathrm{CC} 2$ and CC3 respectively, the corresponding frequency-dependent impedances $\mathbf{Z}_{\mathbf{C C} 1}, \mathbf{Z}_{\mathbf{C C} 2}$ and $\mathbf{Z}_{\mathbf{C C} 3}$ can be expressed by

$$
\begin{aligned}
& \boldsymbol{Z}_{\boldsymbol{C C 1}}=\frac{a_{3} \cdot\left(\left.\frac{V_{\mathbf{1}}}{V_{\mathbf{2}}}\right|_{C C 1}\right)-a_{2}}{-\left.\frac{V_{\mathbf{1}}}{V_{2}}\right|_{C C 1}+a_{1}} \\
& \boldsymbol{Z}_{\boldsymbol{C C 2}}=\frac{a_{3} \cdot\left(\left.\frac{V_{\mathbf{1}}}{V_{\mathbf{2}}}\right|_{C C 2}\right)-a_{2}}{-\frac{V_{\mathbf{1}}}{V_{\mathbf{2}}} \mid C C 2+a_{1}} \\
& \boldsymbol{Z}_{\boldsymbol{C C} \mathbf{3}}=\frac{a_{3} \cdot\left(\left.\frac{V_{1}}{V_{2}}\right|_{C C 3}\right)-a_{2}}{-\left.\frac{V_{\mathbf{1}}}{V_{\mathbf{2}}}\right|_{C C 3}+a_{1}}
\end{aligned}
$$

where $\frac{\boldsymbol{v}_{\mathbf{1}}}{\left.\boldsymbol{V}_{\mathbf{2}}\right|_{C C i}}(\mathrm{i}=1,2$ or 3$)$ can be established straight utilizing the TPMS. Reordering the above equations, they can be expressed as

$$
\begin{aligned}
& Z_{C C 1} \cdot a_{1}+a_{2}-\left(\left.\frac{V_{1}}{V_{2}}\right|_{C C 1}\right) \cdot a_{3}=\left(\left.\frac{V_{1}}{V_{2}}\right|_{C C 1}\right) \cdot Z_{C C 1} \\
& Z_{C C 2} \cdot a_{1}+a_{2}-\left(\left.\frac{V_{1}}{V_{2}}\right|_{C C 2}\right) \cdot a_{3}=\left(\left.\frac{V_{1}}{V_{2}}\right|_{C C 2}\right) \cdot Z_{C C 2} \\
& Z_{C C 3} \cdot a_{1}+a_{2}-\left(\left.\frac{V_{1}}{V_{2}}\right|_{C C 3}\right) \cdot a_{3}=\left(\left.\frac{V_{1}}{V_{2}}\right|_{C C 3}\right) \cdot Z_{C C 3}
\end{aligned}
$$

and in their corresponding matrix form, are given by

$$
\left[\begin{array}{lll}
Z_{C C 1} & 1 & -\left.\frac{V_{1}}{V_{2}}\right|_{C C 1} \\
Z_{C C 2} & 1 & -\left.\frac{V_{1}}{V_{2}}\right|_{C C 2} \\
Z_{C C 3} & 1 & -\left.\frac{V_{1}}{V_{2}}\right|_{C C 3}
\end{array}\right] \times\left[\begin{array}{l}
a_{1} \\
a_{2} \\
a_{3}
\end{array}\right]=\left[\begin{array}{l}
\left.\frac{V_{1}}{V_{2}}\right|_{C C 1} \cdot Z_{C C 1} \\
\left.\frac{V_{1}}{V_{2}}\right|_{C C 2} \cdot Z_{C C 2} \\
\left.\frac{V_{1}}{V_{2}}\right|_{C C 3} \cdot Z_{C C 3}
\end{array}\right]
$$

Using Cramer's rule to solve the above matrix, $\mathrm{a}_{1}, \mathrm{a}_{2}$ and $\mathrm{a}_{3}$ can be expressed by 


$$
\begin{gathered}
a_{1}=\frac{1}{\gamma} \cdot \operatorname{det}\left[\begin{array}{lll}
\left.\frac{V_{1}}{V_{2}}\right|_{C C 1} \cdot Z_{C C 1} & 1 & -\left.\frac{V_{1}}{V_{2}}\right|_{C C 1} \\
\left.\frac{V_{1}}{V_{2}}\right|_{C C 2} \cdot Z_{C C 2} & 1 & -\left.\frac{V_{1}}{V_{2}}\right|_{C C 2} \\
\left.\frac{V_{1}}{V_{2}}\right|_{C C 3} \cdot Z_{C C 3} & 1 & -\left.\frac{V_{1}}{V_{2}}\right|_{C C 3}
\end{array}\right] \\
a_{2}=\frac{1}{\gamma} \cdot \operatorname{det}\left[\begin{array}{lll}
Z_{C C 1} & \left.\frac{V_{1}}{V_{2}}\right|_{C C 1} \cdot Z_{C C 1} & -\left.\frac{V_{1}}{V_{2}}\right|_{C C 1} \\
Z_{C C 2} & \left.\frac{V_{1}}{V_{2}}\right|_{C C 2} \cdot Z_{C C 2} & -\left.\frac{V_{1}}{V_{2}}\right|_{C C 2} \\
Z_{C C 3} & \left.\frac{V_{1}}{V_{2}}\right|_{C C 3} \cdot Z_{C C 3} & -\left.\frac{V_{1}}{V_{2}}\right|_{C C 3}
\end{array}\right] \\
a_{3}=\frac{1}{r} \cdot \operatorname{det}\left[\begin{array}{llll}
Z_{C C 1} & 1 & \left.\frac{V_{1}}{V_{2}}\right|_{C C 1} \cdot Z_{C C 1} \\
Z_{C C 2} & 1 & \left.\frac{V_{1}}{V_{2}}\right|_{C C 2} \cdot Z_{C C 2} \\
Z_{C C 3} & 1 & \left.\frac{V_{1}}{V_{2}}\right|_{C C 3} \cdot Z_{C C 3}
\end{array}\right]
\end{gathered}
$$

Where "det" stands for the determinant of the matrix, and

$$
\gamma=\operatorname{det}\left[\begin{array}{ccc}
Z_{C C 1} & 1 & -\left.\frac{V_{1}}{V_{2}}\right|_{C C 1} \\
Z_{C C 2} & 1 & -\left.\frac{V_{1}}{V_{2}}\right|_{C C 2} \\
Z_{C C 3} & 1 & -\left.\frac{V_{1}}{V_{2}}\right|_{C C 3}
\end{array}\right]
$$

Hence, the final expressions for $\mathrm{a}_{1}, \mathrm{a}_{2}$ and $\mathrm{a}_{3}$ respectively are

$$
\begin{aligned}
& a_{1}=\left[\left.\left.\left(\frac{V_{1}}{V_{2}}\right)\right|_{C C 1} \cdot\left(\frac{V_{1}}{V_{2}}\right)\right|_{C C 2} \cdot\left(Z_{C C 1}-Z_{C C 2}\right)+\left.\left.\left(\frac{V_{1}}{V_{2}}\right)\right|_{C C 1} \cdot\left(\frac{V_{1}}{V_{2}}\right)\right|_{C C 3} \cdot\left(Z_{C C 3}-\right.\right. \\
& \left.\left.Z_{C C 1}\right)\left.\left.\left(\frac{V_{1}}{V_{2}}\right)\right|_{C C 2} \cdot\left(\frac{V_{1}}{V_{2}}\right)\right|_{C C 3} \cdot\left(Z_{C C 2}-Z_{C C 3}\right)\right] / \Lambda \\
& a_{2}=\left[\left.\left.\left(\frac{V_{1}}{V_{2}}\right)\right|_{C C 1} \cdot\left(\frac{V_{1}}{V_{2}}\right)\right|_{C C 2} \cdot Z_{C C 3} \cdot\left(Z_{C C 2}-Z_{C C 1}\right)+\left.\left.\left(\frac{V_{1}}{V_{2}}\right)\right|_{C C 1} \cdot\left(\frac{V_{1}}{V_{2}}\right)\right|_{C C 3} \cdot Z_{C C 2} \cdot\left(Z_{C C 1}-Z_{C C 3}\right)+\right. \\
& \left.\left.\left.\left(\frac{V_{1}}{V_{2}}\right)\right|_{C C 2} \cdot\left(\frac{V_{1}}{V_{2}}\right)\right|_{C C 3} \cdot Z_{C C 1} \cdot\left(Z_{C C 3}-Z_{C C 2}\right)\right] / \Lambda \\
& a_{3}=\left[\left.\left(\frac{V_{1}}{V_{2}}\right)\right|_{C C 1} \cdot Z_{C C 1} \cdot\left(Z_{C C 2}-Z_{C C 3}\right)+\left.\left(\frac{V_{1}}{V_{2}}\right)\right|_{C C 2} \cdot Z_{C C 2} \cdot\left(Z_{C C 3}-Z_{C C 1}\right)+\left.\left(\frac{V_{1}}{V_{2}}\right)\right|_{C C 3} \cdot Z_{C C 3} \cdot\left(Z_{C C 1}-\right.\right. \\
& \left.\left.Z_{C C 2}\right)\right] / \Lambda
\end{aligned}
$$

Where,

$$
\Lambda=\left.\left(\frac{V_{1}}{V_{2}}\right)\right|_{C C 1} \cdot\left(Z_{C C 3}-Z_{C C 2}\right)+\left.\left(\frac{V_{1}}{V_{2}}\right)\right|_{C C 2} \cdot\left(Z_{C C 1}-Z_{C C 3}\right)+\left.\left(\frac{V_{1}}{V_{2}}\right)\right|_{C C 3} \cdot\left(Z_{C C 2}-Z_{C C 1}\right)
$$

After deriving the values of $a_{1}, a_{2}$ and $a_{3}, Z_{X}$ can be found with the measured values of $\boldsymbol{V}_{\mathbf{1}}$ and $\boldsymbol{V}_{\mathbf{2}}$ with the use of the TPMS. Finally, the online impedance of the DUT can be obtained by extracting $\left(\mathbf{Z}_{\mathbf{S}}+\mathbf{Z}_{\mathbf{w}}\right)$ from $\mathbf{Z}_{\mathbf{X}}$.

\section{Section IV. Experimental Results and Validation}

Figure 11 shows the actual implementation of the inductively coupled two-probe measurement setup with improved calibration configuration. As the effectiveness of the calibration procedure holds a great influence on the precision and accuracy of the measured impedance, thus, a $0.1 \Omega, 50 \Omega$ and a $1 \mathrm{k} \Omega$ resistive load are employed in the calibration procedure as calibration standards as shown in Figure 12. The computer-controlled signal generation and acquisition system (SGAS) is a hardware which utilises the National Instruments (NI) PXI interface. A signal generation card (SGC), dual-channel signal acquisition card (SAC) and an embedded controller for the PXI system embodies the SGAS as shown in Figure 13. Two clamp-on inductive probes are employed as the injecting inductive probe (IIP) and the receiving inductive probe (RIP), respectively. It is worth bearing in mind that it is necessary to consider the maximum current flowing through the device under test (DUT) when choosing the inductive probes, so as to prevent the probes from reaching its core saturation. The information of the instruments employed in the calibration configuration are listed in Table 1. 


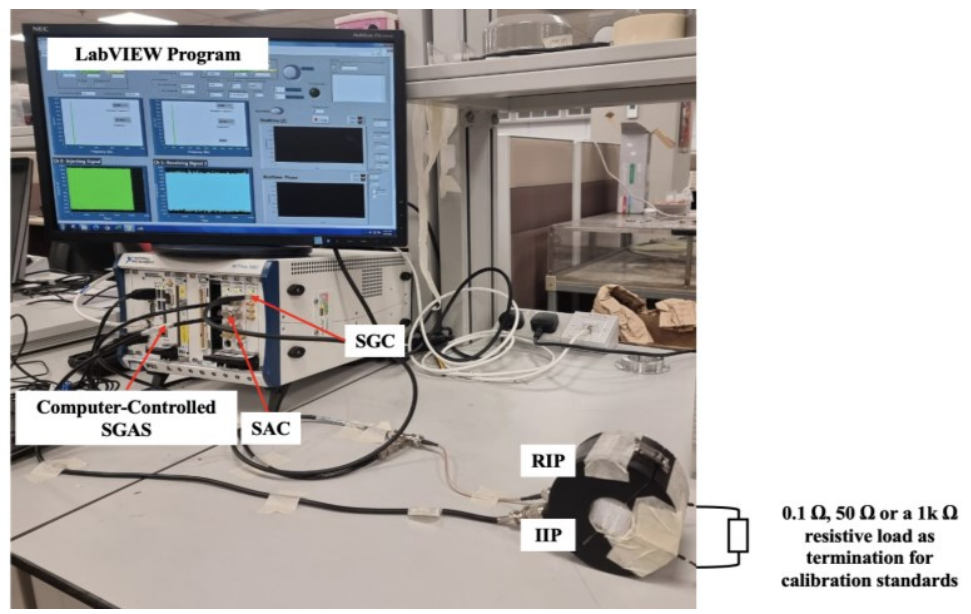

Figure 11. Impedance Measurement setup and calibration configuration for substantiation.

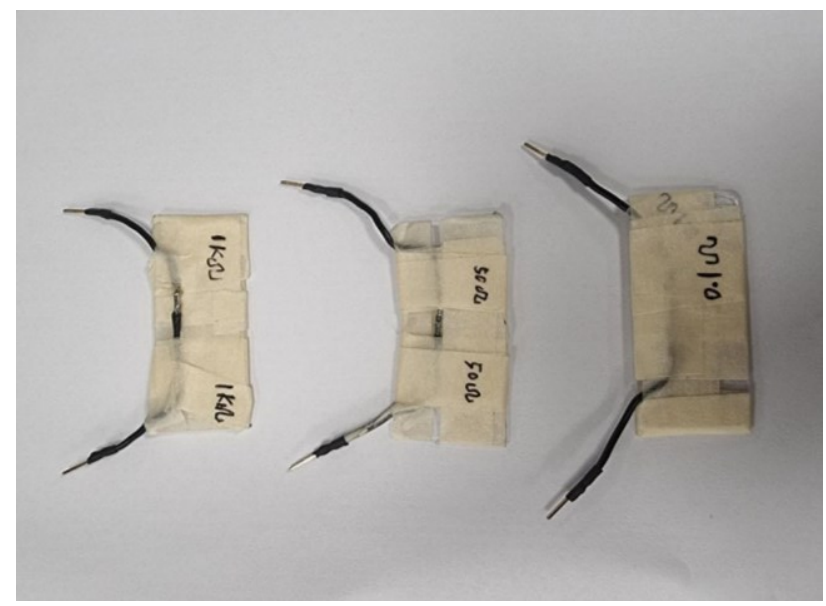

Figure $12.0 .1 \Omega, 50 \Omega$ and a $1 \mathrm{k} \Omega$ resistive load employed in the pre-measurement calibration procedure as shown in Figure 11.

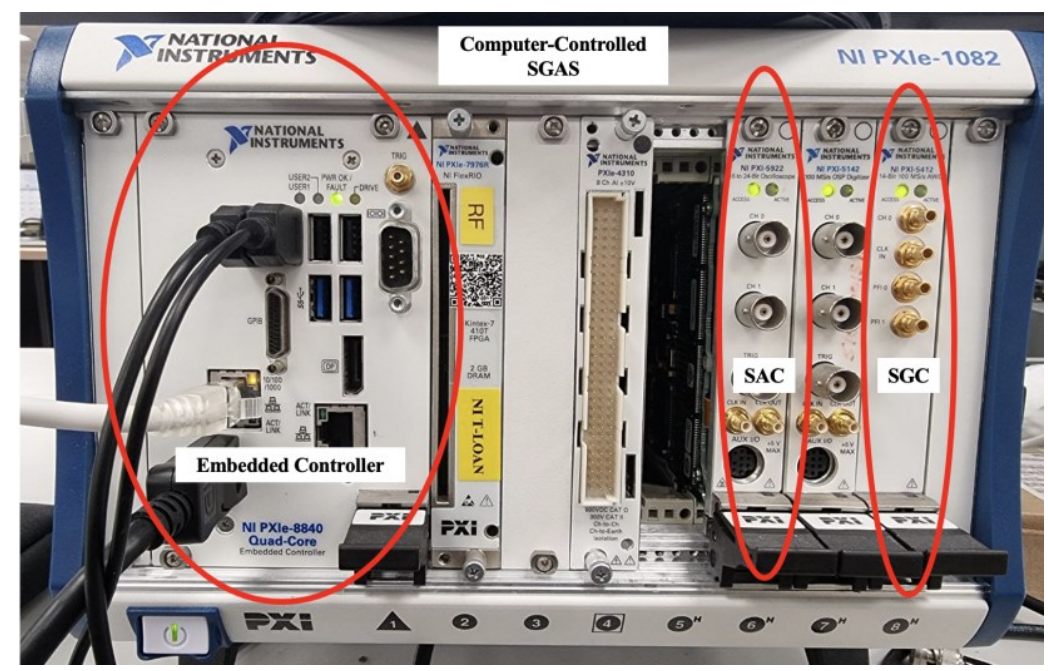

Figure 13. A close-up on the computer-controlled SGAS.

Table 1. Instruments that embodies the calibration configuration. 


\begin{tabular}{cc}
\hline Instrument & Descriptions \\
\hline$S G A S$ & NI PXIe-1082 \\
$S G C$ & NI PXI-5412 \\
$S A C$ & NI PXI-5922 \\
$I I P$ & Solar 9144-1N \\
RIP & Solar 9134-1 \\
Embedded Controller & PXIe-8840 \\
\hline \hline
\end{tabular}

On the basis of the measurement setup, a total of three DUTs namely two through-hole resistors $(15 \Omega, 110 \Omega)$ and one film capacitor $(1 \mathrm{nF})$ are used to run a number of tests. The impedance of each DUT is measured at 19 different frequencies, from $100 \mathrm{kHz}$ to $10 \mathrm{MHZ}$. Through the two-probe measurement setup (TPMS), followed by the proposed improved calibration procedure and the prevailing OSL calibration procedure, the online impedances of each DUT are extracted and compared against the reference values obtained from the impedance analyzer. The calibration criterions for the prevailing OSL calibration are as follow: a $0.1 \mathrm{~m}$ copper wire left opened, $0.1 \Omega$

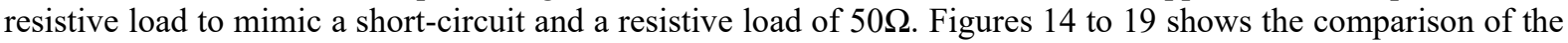
obtained online impedances $\left(\mathbf{Z}_{\mathbf{x}}\right)$ on the basis of the proposed improved calibration procedure and the prevailing OSL calibration procedure.

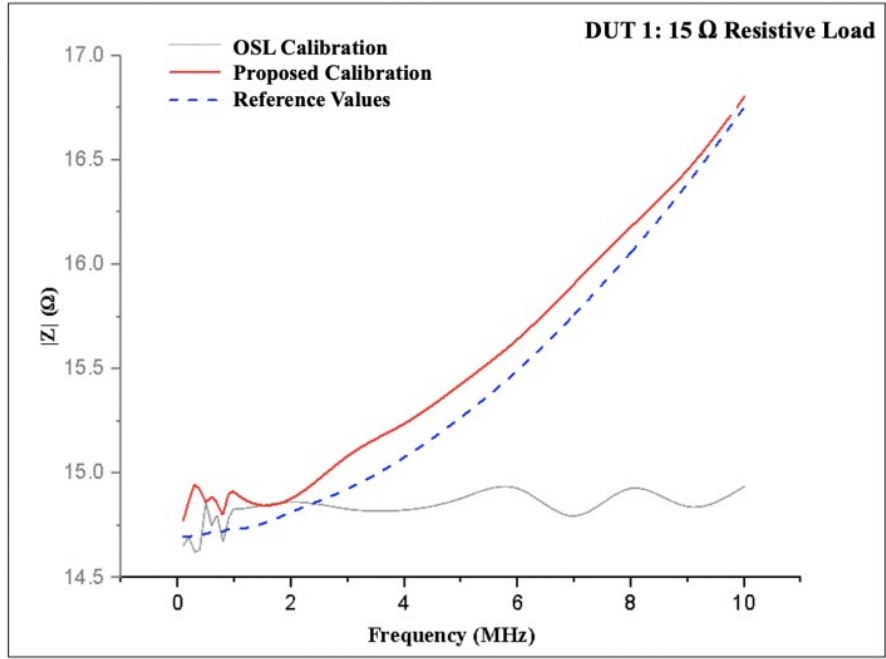

Figure 14. Extracted Online Impedance (Magnitude) of DUT 1: 15 $\Omega$ using the proposed calibration procedure compared with the existing OSL calibration procedure.

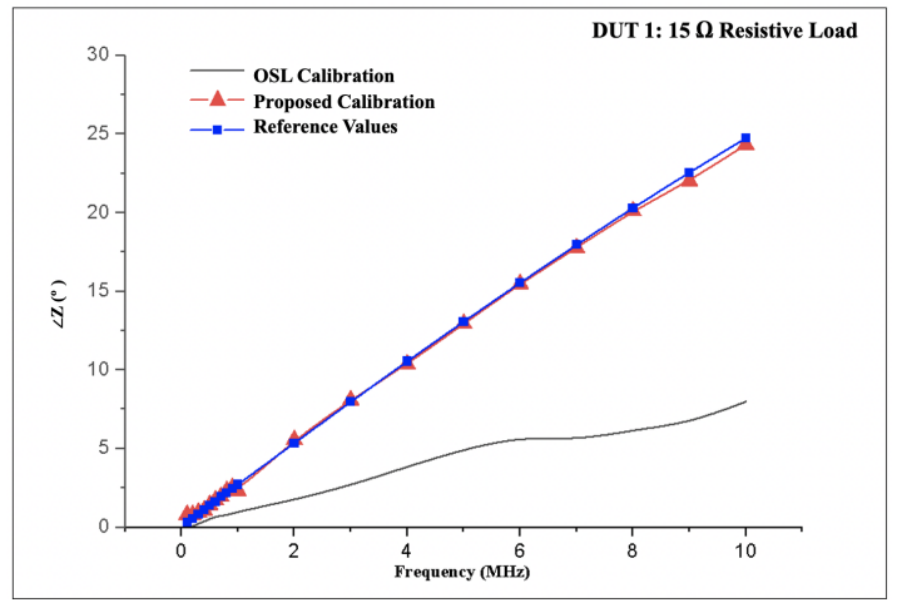

Figure 15. Extracted Online Impedance (Phase) of DUT 1: $15 \Omega$ using the proposed calibration procedure compared with the existing OSL calibration procedure. 


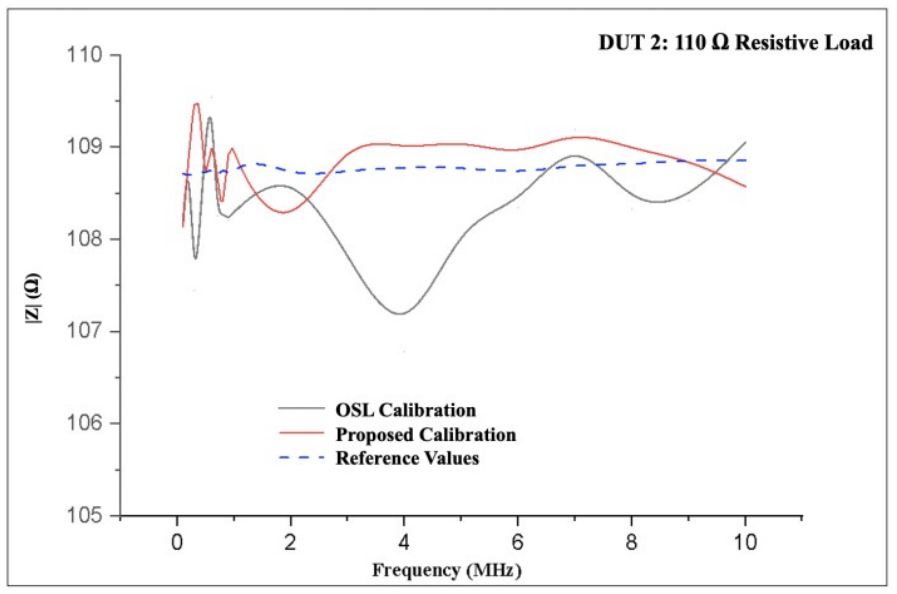

Figure 16. Extracted Online Impedance (Magnitude) of DUT 2: $110 \Omega$ using the proposed calibration procedure compared with the existing OSL calibration procedure.

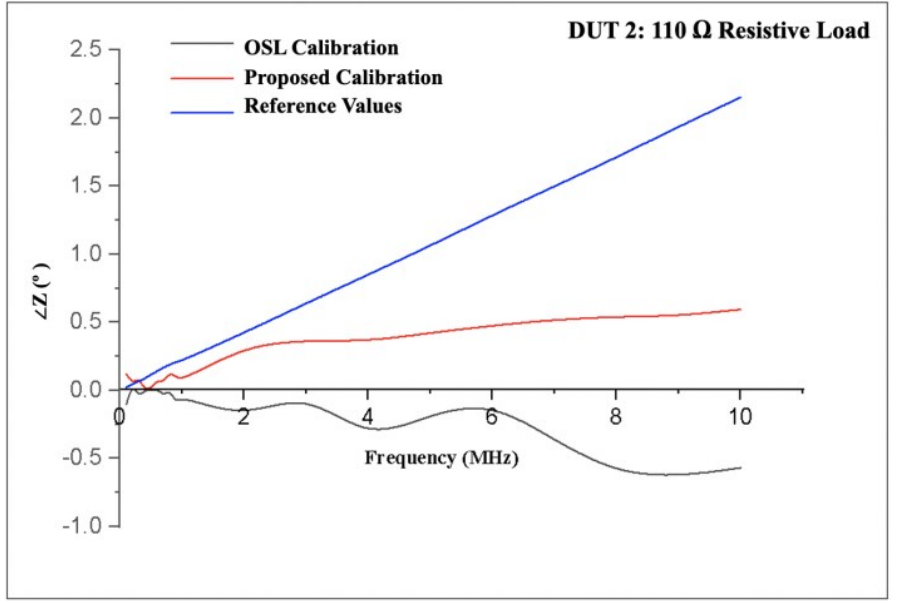

Figure 17. Extracted Online Impedance (Phase) of DUT 2: $110 \Omega$ using the proposed calibration procedure compared with the existing OSL calibration procedure.

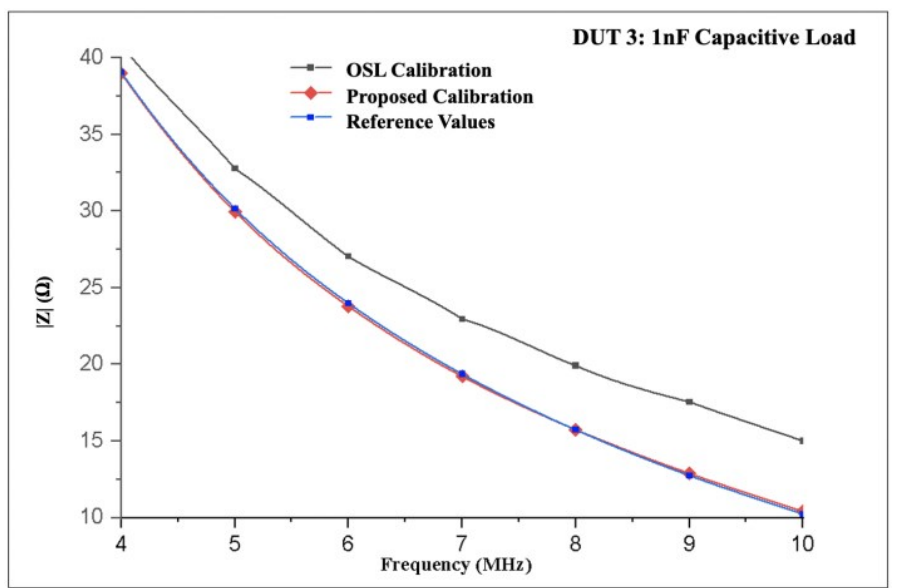

Figure 18. Extracted Online Impedance (Magnitude) of DUT 3: 1nF using the proposed calibration procedure compared with the existing OSL calibration procedure. 


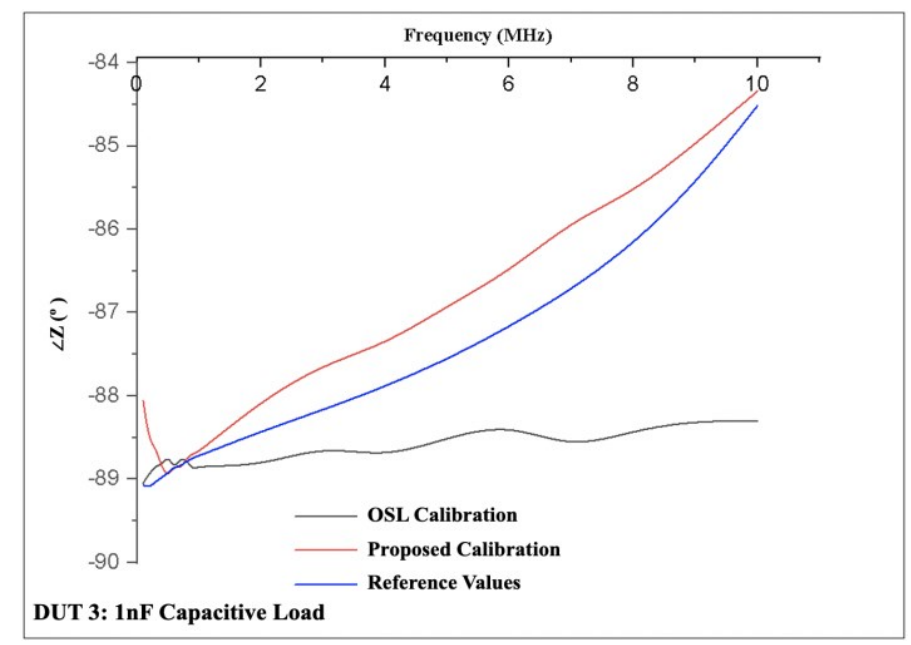

Figure 19. Extracted Online Impedance (Phase) of DUT 3: 1nF using the proposed calibration procedure compared with the existing OSL calibration procedure.

As seen in Figure 13 to Figure 19, the deviations through the medium of the proposed improved calibration and the prevailing OSL calibration procedure are rather insignificant for all DUTs when the range of the frequency is below $1 \mathrm{MHz}$. This is for the reason that the influence the residual capacitances and inductances hold are insignificant for frequency below $1 \mathrm{MHz}$, thus it can be neglected in low frequency levels. Conversely, the deviations of the online impedance measured using the prevailing OSL calibration procedure starts to increase along with the increasing frequency of the injecting test signal $\left(f_{\text {sig }}\right)$. When the frequency of $f_{\text {sig }}$ is comparably large, for example, above $1 \mathrm{MHz}$, the OSL calibration criterions diverge from the corresponding optimal values, which are $\infty, 0$ and $50 \Omega \angle 0^{\circ}$, which thus refuses the assumption in consequence. In essence, it is validated that the prevailing OSL calibration will result in considerable inaccuracy in the extracted values of the online impedances in high frequencies systems. On the other hand, if the frequency-dependent characteristics of the calibration criterions are factored in and developed on the proposed improved calibration procedure, the accuracy and precision of the extracted online impedances enhanced notably.

\section{Section V. Conclusion}

In view of the significance and influence the online impedance has on many vital electrical systems, an extensive literature review on the prevailing online impedance extraction methods has been carefully explored. Taking into account the constraints and drawbacks of the existing methods, this paper has introduced an improved and optimized calibration procedure for the inductive coupling impedance measurement setup. First off, by utilizing a computer-controlled signal generation and acquisition system (SGAS) and two clamp-on inductive probes, namely the injecting inductive probe (IIP) and the receiving inductive probe (RIP) that embodies the fundamental structure of the measurement setup, this improved calibration measurement setup is capable of extracting the online impedance of critical electrical assets more precisely and accurately.

In practical situations, there may be space constraints which thus inevitably reduce the distance between the two probes, which may induce a coupling effect which may affect the integrity of the measured impedance to a certain extent. On the basis of the three-port network theory, an extensive circuit model of the proposed improved calibration measurement setup was introduced in Section III, where the influence of the probe-to-probe coupling between the two fore mentioned inductive probes should be taken into consideration. The improved calibration procedure is proposed to eliminate any ramifications of the probe-to-probe coupling, which further increase the reliability and accuracy of the impedance measurement procedure. Hence, with the proposed improved calibration, the online impedance measurement procedure will still be able to uphold its accuracy and integrity despite the influence of the probe-to-probe coupling. Additionally, by integrating surge protection modules into the inductive coupling measurement configuration, the system is less prone to unpredicted power surges, and thus minimizing the risk of irreparable damages. Thence, it broadens the purview of implementation of this method to electrical systems with notable power surges. 


\section{References}

[1] Z. Zhao, "Measurement setup consideration and implementation for inductively coupled online impedance extraction," Ph.D. Thesis, School of Electrical and Electronic Engineering, Nanyang Technological University, Singapore, 2021, doi: 10.32657/10356/146738.

[2] S. Cobreces, E. J. Bueno, D. Pizarro, F. J. Rodriguez, and F. Huerta, "Grid impedance monitoring system for distributed power generation electronic interfaces," IEEE Trans. Instrum. Meas., vol. 58, no. 9, pp. 31123121, Sep. 2009, doi: 10.1109/TIM.2009.2016883.

[3] Y. He, S.-H. Chung, C.-T. Lai, X. Zhang, and W. Wu, "Active cancelation of equivalent grid impedance for improving stability and injected power quality of grid-connected inverter under variable grid condition," IEEE Trans. Power Electron., vol. 33, no. 11, pp. 9387-9398, Nov. 2018, doi: 10.1109/TPEL.2018.2793459.

[4] L. Asiminoaei, R. Teodorescu, F. Blaabjerg, and U. Borup, "A new method of on-line grid impedance estimation for PV inverter," in Proc. IEEE Appl. Power Electron. Conf. Expo. (APEC), Anaheim, CA, USA, 2004, pp. 1527-1533, doi: 10.1109/APEC.2004.1296067.

[5] Z. Zhao et al., "Online insulation fault detection of stator winding of induction motor based on a nonintrusive impedance extraction technique," in Proc. IEEE Int. Conf. Intelligent Rail Transportation (ICIRT), Singapore, 2018, pp. 1-5, doi: 10.1109/ICIRT.2018.8641627.

[6] Z. Zhao, F. Fan, W. Wang, Y. Liu, and K. Y. See, "Detection of stator interturn short-circuit faults in inverter-fed induction motors by online common-mode impedance monitoring," IEEE Trans. Instrum. Meas., vol. 70, 2021, Art no. 3513110, doi: 10.1109/TIM.2021.3066193.

[7] Z. Zhao, A. Weerasinghe, W. Wang, E. K. Chua, and K. Y. See, "Eliminating the effect of probe-to-probe coupling in inductive coupling method for in-circuit impedance measurement," IEEE Trans. Instrum. Meas., vol. 70, 2021, Art no. 1000908, doi: 10.1109/TIM.2020.3013688.

[8] L. Asiminoaei, R. Teodorescu, F. Blaabjerg, and U. Borup, "Implementation and test of an online embedded grid impedance estimation technique for PV inverters," IEEE Trans. Ind. Electron., vol. 52, no. 4, pp. 11361144, Aug. 2005, doi: 10.1109/TIE.2005.851604.

[9] M. Sumner, B. Palethorpe, and D. Thomas, "Impedance measurement for improved power quality-part 1: The measurement technique," IEEE Trans. Power Del., vol. 19, no. 3, pp. 1442-1448, Jul. 2004, doi: 10.1109/TPWRD.2004.829873.

[10] A. V. Timbus, R. Teodorescu, F. Blaabjerg, and U. Borup, "Online grid measurement and ENS detection for PV inverter running on highly inductive grid," IEEE Power Electron. Lett., vol. 2, no. 3, pp. 77-82, Sep. 2004, doi: 10.1109/LPEL.2004.834921.

[11] N. Hoffmann and F. W. Fuchs, "Minimal invasive equivalent grid impedance estimation in inductiveresistive power networks using Extended Kalman Filter," IEEE Trans. Power Electron., vol. 29, no. 2, pp. 631-641, Feb. 2014, doi: 10.1109/TPEL.2013.2259507.

[12] W. Huang and J. A. A. Qahouq, "An online battery impedance measurement method using dc-dc power converter control," IEEE Trans. Ind. Electron., vol. 61, no. 11, pp. 5987-5995, Nov. 2014, doi: 10.1109/TIE.2014.2311389.

[13] D. K. Alves, R. Ribeiro, F. B. Costa, and T. Rocha, "Real-time wavelet-based grid impedance estimation method," IEEE Trans. Ind. Electron., vol. 66, no. 10, pp. 8263-8265, Oct. 2019, doi: 10.1109/TIE.2018.2870407.

[14] C. González, J. Pleite, V. Valdivia, and J. Sanz, "An overview of the on line application of frequency response analysis (FRA)," in Proc. IEEE Int. Symp. Ind. Electron., Vigo, Spain, 2007, pp. 1294-1299, doi: 10.1109/ISIE.2007.4374785.

[15] T. Funaki, N. Phankong, T. Kimoto, and T. Hikihara, "Measuring terminal capacitance and its voltage dependency for high-voltage power devices," IEEE Trans. Power Electron., vol. 24, no. 6, pp. 1486-1493, Jun. 2009, doi: 10.1109/TPEL.2009.2016566.

[16] X. Shang, D. Su, H. Xu, and Z. Peng, "A noise source impedance extraction method for operating SMPS using modified LISN and simplified calibration procedure," IEEE Trans. Power Electron., vol. 32, no. 6, pp. 4132-4139, Jun. 2017, doi: 10.1109/TPEL.2016.2631578. 
[17] R. A. Southwick and W. C. Dolle, "Line impedance measuring instrumentation utilizing current probe coupling," IEEE Trans. Electromagn. Compat., vol. EMC-13, no. 4, pp. 31-36, Nov. 1971, doi: 10.1109/TEMC.1971.303150.

[18] K. Li, K. Y. See, and X. Li, "Inductive coupled in-circuit impedance monitoring of electrical system using two-port ABCD network approach," IEEE Trans. Instrum. Meas., vol. 64, no. 9, pp. 2489-2495, Sep. 2015, doi: 10.1109/TIM.2015.2403091.

[19] S. B. Rathnayaka, K. Y. See, and K. Li, "On-line impedance monitoring of transformer based on inductive coupling approach," IEEE Trans. Dielectr. Electr. Insul., vol. 24, no. 2, pp. 1273-1279, Apr. 2017, doi: 10.1109/TDEI.2017.006111.

[20] Z. Zhao, K.-Y. See, E.-K. Chua, A. S. Narayanan, A. Weerasinghe, and W. Chen, "Extraction of voltagedependent capacitances of SiC device through inductive coupling method," in Joint Proc. IEEE Int. Symp. Electromagn. Compat. and Proc. Asia-Pacific Int. Symp. Electromagn. Compat. (Joint EMC \& APEMC), Singapore, 2018, pp. 1301-1304, doi: 10.1109/ISEMC.2018.8393999.

[21] A. S. Narayanan et al., "Study on the parameters affecting the impedance extraction accuracy by inductive coupling method," in Proc. IEEE Int. Conf. Intelligent Rail Transportation (ICIRT), Singapore, 2018, pp. 15, doi: 10.1109/ICIRT.2018.8641624.

[22] Z. Zhao et al., "Voltage-dependent capacitance extraction of SiC power MOSFETs using inductively coupled in-circuit impedance measurement technique," IEEE Trans. Electromagn. Compat., vol. 61, no. 4, pp. 1322-1328, Aug. 2019, doi: 10.1109/TEMC.2019.2914704.

[23] C. J. Kikkert, "An on-line plc frequency impedance analyzer," in Proc. IEEE Int. Conf. Smart Gird Comm., Vancouver, BC, Canada, 2013, pp. 606-611, doi: 10.1109/SmartGridComm.2013.6688025.

[24] D. M. Pozar, Microwave engineering. Hoboken, NJ, USA: Wiley, 2012.

[25] Z. Zhao, K. Y. See, E. K. Chua, A. S. Narayanan, W. Chen, and A. Weerasinghe, "Time-variant in-circuit impedance monitoring based on the inductive coupling method," IEEE Trans. Instrum. Meas., vol. 68, no. 1, pp. 169-176, Jan. 2019, doi: 10.1109/TIM.2018.2838198. 